\title{
Rearview
}

\section{Will Open RAN remain open?}

\section{A regular column on the information industries}

\section{Peter Curwen and Jason Whalley}

Peter Curwen is Independent Scholar based in Leeds, UK. Jason Whalley is based at the Newcastle Business School, Northumbria University, Newcastle upon Tyne, UK.

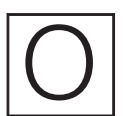

ver the past several decades, the number of equipment vendors capable of rolling out mobile networks has shrunk noticeably, such that the market is now dominated by Huawei which has taken advantage of its near monopoly of its immense home market where ZTE is also very active Ericsson and Nokia.

From a network operator's viewpoint, this matters because once a basic $2 \mathrm{G}$ network has been commissioned from a specific vendor, it is inevitably easier and more efficient to contract with the same vendor for $3 \mathrm{G}$ and $4 \mathrm{G}$ infrastructure that can replace and/or build on the existing network without engendering issues of compatibility. In effect, therefore, operators have effectively locked themselves into their relationships with vendors, even though it is possible that there is a cheaper alternative on offer to upgrade.

$5 G$, as is being rolled out in its NonStandalone version - that is, as an upgrade to an existing $4 \mathrm{G}$ network - would accordingly appear to simply be a continuation of what has gone before. However, there are two further factors to consider.

The first of these is that the U.S. Government has determined that
Huawei equipment must be stripped from domestic mobile networks, with many other countries such as the UK following suit. The UK has sought to diversify the telecommunications value chain, opening discussions with other equipment suppliers and establishing a taskforce to explore how more companies could be encouraged to enter the market. But it is by no means certain that these efforts will be successful, and even if they are, it will be some time before new players are able to enter the market. This has effectively opened up opportunities for Ericsson and Nokia to become the leading vendors of choice for $5 \mathrm{G}$.

But this leads on to the second point, which is that operators will remain locked into an even smaller number of vendors, leaving the latter able, in principle, to raise prices without fear of being undercut.

Being locked in does, however, have certain advantages compared to a multi-vendor open system. As T-Mobile has noted, one advantage of having a complete solution provided by a single vendor is that if anything goes wrong, the operator knows who to turn to for help. 
Not surprisingly, other, much smaller, vendors are proposing a way out of this dilemma, especially in relation to the equipment needed for Standalone $5 \mathrm{G}$ - that is, for new networks that are wholly independent of existing infrastructure and operate in much higher bandwidths.

Their solution, commonly known as Open Radio Access Network (Open RAN) - alternatively, Open virtual RAN or Open Radio-Access Network - is very much a work in progress. The underlying point, as noted above, is that a mobile network can only function at present provided all the hardware can communicate, which means that the hardware itself and the software that underpins it must be provided by the same vendor. With Open RAN, standardised hardware can be purchased to replace bespoke units from a single vendor. This potentially increases the resilience of a network and, through enhanced competition between suppliers, puts downwards pressure on costs. The O-RAN Alliance, for one, has been working to develop Open RAN, but a group of companies including operators AT\&T, China Mobile and Deutsche Telekom, as well as the likes of Facebook, Google and Intel has recently set out to expedite development via the Open Networking Foundation - see www. opennetworking.org - which is building an open source Near Real-Time RAN Intelligent Controller (NRT-RIC) compatible with the O-RAN architecture.

The difficulty remains that open source means strictly that that there should be no demands for royalties from the developer community. However, the Telecom Infra Project, which is underwritten by Facebook, do not always adhere to the principle of granting licensing on reasonable, fair and nondiscriminatory (RAND) terms. The O-RAN Alliance is also far from royalty-free. It has been suggested that confusion can arise because of the mistaken assumption that open source and Open RAN are one and the same thing.

It is evident that it is much easier for an operator starting from scratch, such as Rakuten in Japan, to introduce Open RAN compared to incumbents that own large numbers of existing sites with their installed equipment. It is also evident that Ericsson and Nokia have been offering big discounts on $5 \mathrm{G}$ equipment to their major customers to keep them locked in while Huawei loomed on the horizon - the evidence for this to be found in the low operating margins revealed in their recent financial reports - and can continue to do so if Open RAN posed a serious threat.

Various reports have appeared that assess the prospects for Open RAN. In February 2019, for example, $5 \mathrm{G}$ Americas produced an initial White Paper that considered the prospects for open source in relation to $5 \mathrm{G}$. It followed up in November 2020 with a further White Paper that highlighted key architecture aspects focused on disaggregating software from hardware and open interfaces to allow multi-vendor interoperability. After a brief review of the ecosystem bodies involved with Open RAN, it went on to enumerate the trials and deployments taking place and to examine the motivations and challenges faced by operators wishing to switch to Open RAN. Finally, it examined the role of artificial intelligence and machine learning initiatives in enabling Open RAN architectures and associated functions for selforganizing networks, management, orchestration and automation to meet the variety of use cases for $5 \mathrm{G}$ and beyond.

Understandably, operators find themselves attracted to the idea of a multi-vendor world where everyone produces compatible equipment. For example, the use of Open RAN by Vodafone in Ireland, commencing in November 2020, is potentially significant, especially as it will shed some light on whether an Open RAN network can, as its supporters claim, be rolled out much more cheaply than a network using the equipment supplied by the major vendors. Vodafone has also reacted to the government's ban on the installation of Huawei's products in the UK by committing to replace Huawei with Open RAN at roughly 2,600 radio masts.

But incumbent operators are mostly not rushing to join in, partly because it has been estimated that the RAN only comprises roughly $20 \%$ of total capital expenditure, which is itself only a fraction of an operator's total costs, so a switch to Open RAN will not effect a massive saving for an operator.

The likes of Deutsche Telekom and Orange have so far failed to specify how many base stations where Open RAN will be deployed - although Vodafone in the UK and Telefónica in Germany have made a modest commitment to installing Open RAN on their respective networks.

Meanwhile, with contracts already signed to acquire $5 \mathrm{G}$ products from mainstream vendors, there is little money left to allocate to smaller Open RAN vendors.

It is of interest that the main European operators have turned to the EU for subsidies, playing upon EU fears about Chinese vendors, but that is awkward when Open RAN equipment is supplied almost entirely by non-EU companies and the operators are in good financial health.

In February 2021, DoCoMo began to supply inter-operable Open RAN products in alliance with Rakuten and the likes of Mavenir, which could be described as an ecosystem within an ecosystem - in other words, it manifested a desire to overcome the 
sheer hard work involved in putting together a network where the components had to be sourced from a variety of providers by combining certain parts into pre-arranged packages with guaranteed interoperability.

But if specific groups are being set up to provide their own version of Open RAN, this could simply be seen as the first step in a vendor lock-in version 2.0 that did not involve the incumbent vendors.

In March, it was noted that whereas there was supposed to have been a coming together around the specifications developed by the O-RAN Alliance, a study of developments by TeckNexus had revealed that none of the Open RAN networks it had recently analysed were O-RAN compliant. The incompatibility of the Rakuten network with the specifications was especially notable. The question was raised as to whether the specifications would ever achieve widespread acceptability. As one commentator has put it, there is one vision but many competing interests.

As noted, the O-RAN Alliance contains members from both the USA (82 at end-2020) and China (44 at end-2020). This causes problems because of the treatment of Huawei and ZTE and the Open RAN Policy Coalition was set up as a body with no Chinese membership as an alternative to the Alliance - see www.openranpolicy.org. Nevertheless, good progress is being made in developing a software-defined RAN (SD-RAN) compatible with the O-RAN architecture.
So where does the above leave us? Firstly, it is evident that Open RAN is still very much in its development stage with few products actually on the market. Secondly, and more importantly, that there are competing industry organisations that are pushing their own agenda even if it conflicts with that of other similar groups. Hence, there are likely to be a number of competing versions of Open RAN with operators forced to adopt one or other version if they wish to avoid being locked in to Ericsson and Nokia - but that is not exactly what Open RAN is supposed to be about.

\section{Corresponding author}

Peter Curwen can be contacted at: pjcurwen@hotmail.com 